\title{
PRIMARY ADRENAL LYMPHOMA
}

\section{Uzma Faruqi, Pawel Kaczmarek, Gleb Ivanov, Benjamin Field East Surrey Hospital}

\section{Case Summary}

A 68 year old man diagnosed with a pulmonary embolus was found to have bilateral adrenal masses (image 1). Whilst awaiting outpatient follow up he was readmitted with pyrexia and fatigue. Repeat CT revealed the adrenal masses had grown significantly, the left in particular (image 2). There was biochemical evidence of adrenal insufficiency. CT guided adrenal biopsy elucidated the diagnosis of a high grade B cell lymphoma and R-CHOP chemotherapy was instituted. There has been an excellent response to treatment with rapid reduction in tumour size (image 3 ).

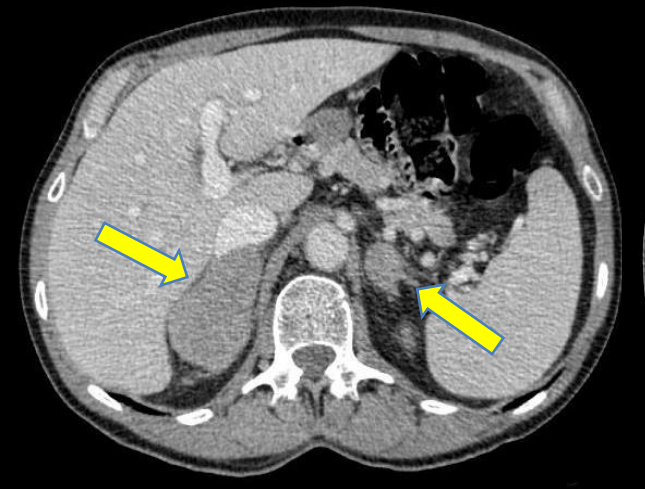

Image 1:

Initial CT following presentation with PE. Incidental finding of adrenal masses as indicated by the arrows.

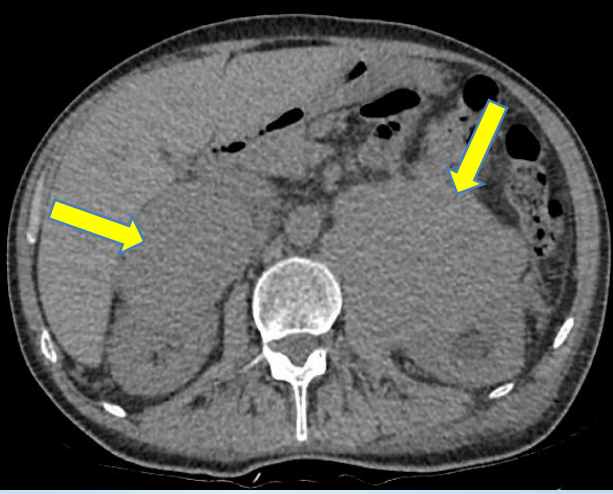

Image 2:

Re-presentation with adrenal insufficiency. Increased size of adrenal masses demonstrated.

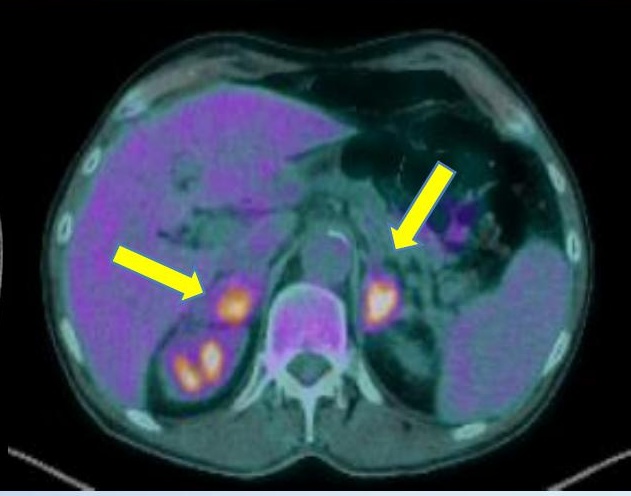

Image 3:

PET CT after initiation of R-CHOP. The metabolically active adrenal masses are significantly smaller.

\section{PRIMARY ADRENAL LYMHPOMA AT A GLANCE}

Diagnosis: lymphoma confirmed on histology +

(i) one or both adrenal glands involved; these are the primary lesions

(ii) no previous history of lymphoma involving other organs

Prevalence: $<200$ reported cases, accounts for $<1 \%$ of all $\mathrm{NHL}$

Gender: male> female

Age: $62 \pm 14$ years

Region: Asia ( $>50 \%)$, Europe $(<30 \%)$

Thought to be associated with:

Immunodeficiency

EBV

P53 and c-kit oncogene mutations
Symptoms: pain, fatigue, pyrexia, weight loss, anorexia

Signs: adrenal insufficiency e.g. buccal/skin hyperpigmentation, may have hepatosplenomegaly or lymphadenopathy

\section{Extra Laboratory tests:}

cortisol/synacthen test, LDH

Imaging modality of choice: PET CT, up to $70 \%$ are bilateral
Histological subtypes: $70 \%$ DLBCL

Treatment: chemotherapy dependent on histology e.g. RCHOP for DLBCL, surgery, radiotherapy

Prognosis: insufficient data Poor prognostic features:

Advanced stage High LDH

Bulky disease

Older age at diagnosis 